\title{
Scaling properties of the dynamics at first-order quantum transitions when boundary conditions favor one of the two phases
}

\author{
Andrea Pelissetto, ${ }^{1}$ Davide Rossini, ${ }^{2}$ and Ettore Vicari ${ }^{2},{ }^{*}$ \\ ${ }^{1}$ Dipartimento di Fisica dell'Università di Roma "La Sapienza" and INFN, Sezione di Roma I, I-00185 Roma, Italy \\ ${ }^{2}$ Dipartimento di Fisica dell'Università di Pisa and INFN, Largo Pontecorvo 3, I-56127 Pisa, Italy
}

(Dated: April 20, 2020)

\begin{abstract}
We address the out-of-equilibrium dynamics of a many-body system when one of its Hamiltonian parameters is driven across a first-order quantum transition (FOQT). In particular, we consider systems subject to fixed boundary conditions, favoring one of the two phases separated by the FOQT: more precisely, boundary conditions that favor the same magnetized phase (EFBC) or opposite phases (OFBC) at the two ends of the chain. These issues are investigated within the paradigmatic one-dimensional quantum Ising model, in which FOQTs are driven by the longitudinal magnetic field $h$. We study the dynamic behavior for an instantaneous quench and for a protocol in which $h$ is slowly varied across the FOQT. We develop a dynamic finite-size scaling theory for both EFBC and OFBC, which displays some remarkable differences with respect to the case of neutral boundary conditions. The corresponding relevant time scale shows a qualitative different size dependence in the two cases: it increases exponentially with the size in the case of EFBC, and as a power of the size in the case of OFBC.
\end{abstract}

\section{INTRODUCTION}

Quantum phase transitions are striking signatures of many-body collective behaviors [1]3]. They are continuous when the ground state of the system changes continuously at the transition point and correlation functions develop a divergent length scale. They are instead of first order when ground-state properties are discontinuous across the transition point. In general, singularities develop only in the infinite-volume limit. If the size $L$ of the system is finite, all properties are analytic as a function of the external parameter driving the transition. However, around the transition point, thermodynamic quantities and large-scale properties develop peculiar scaling behaviors, depending on the general features of the transition. Their understanding is essential to correctly interpret experimental or numerical data, when phase transitions are investigated in relatively small systems - see, e.g., Refs. 4 11. Moreover, their investigation may lead us to discover novel phenomena that emerge in the strongly correlated dynamic regime arising at quantum transitions.

These issues are important not only for continuous quantum transitions, but also for first-order quantum transitions (FOQTs), essentially for two reasons. First, FOQTs are phenomenologically relevant, as they occur in a large number of quantum many-body systems, including quantum Hall samples [12, itinerant ferromagnets [13], heavy fermion metals [14 16], etc. Second, the low-energy properties at FOQTs are particularly sensitive to the boundary conditions, giving rise to a variety of behaviors, that is even wider than at continuous quantum transitions. Indeed, depending on the type of boundary

*Authors are listed in alphabetic order. conditions, for example whether they are neutral or favor one of the phases, the behavior at FOQTs may be characterized by qualitatively different dynamic properties [10, 11, 17, 20, associated with time scales that have an exponential or power dependence on the size of the system.

In this paper we investigate the out-of-equilibrium dynamics of a many-body system undergoing a FOQT, when one of its Hamiltonian parameters is driven across the FOQT. In particular, we study such processes in the presence of boundary conditions that favor one of the two phases separated by the FOQT. This work extends the results presented in Refs. [21, 22], where the dynamic properties of systems with neutral boundary conditions were discussed. As we shall see, notable differences emerge when the system is subject to boundary conditions favoring one of the phases.

We study the above issues within the one-dimensional quantum Ising model in the presence of a transverse field, which provides an optimal theoretical laboratory for the investigation of phenomena emerging at quantum transitions. Indeed its zero-temperature phase diagram presents a line of FOQTs driven by a longitudinal external field $h$, ending at a continuous quantum transition. We focus on the dynamic behavior along the FOQT line, considering boundary conditions that favor one of the two magnetized phases. This is obtained by imposing appropriate boundary conditions: equal fixed boundary conditions (EFBC), meaning that both boundaries favor the same magnetized phase, and opposite fixed boundary conditions (OFBC), meaning that the boundaries favor the phases with opposite magnetization. We are interested in the out-of-equilibrium dynamic behavior arising when a time-dependent longitudinal field $h$ varies across the value $h=0$, associated with the FOQT. For this purpose, we consider two limiting cases: an instantaneous quench from one phase to the other and a time protocol in which $h$ is slowly changed across the FOQT. We 
show that, for both EFBC and OFBC, the system develops a dynamic scaling behavior, as it occurs for neutral boundary conditions [21. However, the dynamic scaling with non-neutral boundary conditions presents peculiar features with respect to those with neutral boundary conditions, making their study necessary to achieve a deep and complete understanding of the phenomenology of FOQTs. Moreover, we anticipate that the dynamic scalings at EFBC and OFBC differ significantly, leading to scaling properties with very different time scales.

It is worth mentioning that analogous issues have been investigated for classical systems undergoing first-order transitions, to understand the dependence of the equilibrium and out-of-equilibrium properties on the boundary conditions - see, e.g., Refs. [23 33].

The paper is organized as follows. In Sec. III we introduce the one-dimensional quantum Ising model, and the dynamic protocols we consider. In Sec. [III we recap the relevant features of the equilibrium finite-size scaling behavior of the Ising chain with EFBC and OFBC. In Sec. IV and Sec. V we discuss the dynamic behavior in the presence of EFBC and OFBC, respectively. Our general arguments are supported by analytical and numerical calculations. Finally, in Sec. VI we summarize our findings and draw our conclusions.

\section{THE QUANTUM ISING CHAIN ALONG THE FIRST-ORDER TRANSITION LINE}

The quantum Ising chain in a transverse field is a paradigmatic quantum many-body system showing continuous and first-order quantum transitions. The Hamiltonian reads

$$
H_{\mathrm{Is}}=-J \sum_{\langle x, y\rangle} \sigma_{x}^{(3)} \sigma_{y}^{(3)}-g \sum_{x} \sigma_{x}^{(1)}-h \sum_{x} \sigma_{x}^{(3)}
$$

where $\boldsymbol{\sigma} \equiv\left(\sigma^{(1)}, \sigma^{(2)}, \sigma^{(3)}\right)$ are the spin-1/2 Pauli matrices, the first sum is over all nearest-neighbor bonds $\langle x, y\rangle$, while the second and the third sums are over the $L$ sites of the chain. We assume $\hbar=1, J=1$, and, without loss of generality, $g>0$. At $g=1$ and $h=0$, the model undergoes a continuous quantum transition belonging to the two-dimensional Ising universality class, separating a disordered phase $(g>1)$ from an ordered $(g<1)$ one. For any $g<1$, the longitudinal field $h$ drives FOQTs along the $h=0$ line.

Here we focus on the dynamic behavior along the FOQT line for $g<1$. In particular, we consider boundary conditions that favor one of the two magnetized phases, EFBC and OFBC. They are obtained by adding fixed spin states at two additional points $x=0$ and $x=L+1$ : for EFBC we fix $|\downarrow\rangle$ at both endpoints $x=0$ and $x=L+1$, while for OFBC we fix $|\downarrow\rangle$ at the endpoint $x=0$ and $|\uparrow\rangle$ at the endpoint $x=L+1$. As we shall see, EFBC and OFBC lead to drastically different dynamic behaviors at the FOQT, characterized by an exponential or a power dependence on the size of the relevant scaling variables, respectively.

The low-energy properties at a FOQT crucially depend on the chosen boundary conditions, even in the $L \rightarrow \infty$ limit - see, e.g., Refs. 10, 11, 17, 18, 34, 35]. If one considers neutral boundary conditions, i.e., boundary conditions that do not favor any of the two phases, in the infinite-volume limit the FOQT is characterized by the crossing of the two states $|+\rangle$ and $|-\rangle$ with opposite longitudinal magnetization, that represent the ground states for $h>0$ and $h<0$, respectively. Correspondingly, the magnetization is discontinuous at $h=0$ [36],

$$
\lim _{h \rightarrow 0^{ \pm}} \lim _{L \rightarrow \infty}\left\langle \pm\left|\sigma_{x}^{(3)}\right| \pm\right\rangle= \pm m_{0}, \quad m_{0}=\left(1-g^{2}\right)^{1 / 8} .
$$

In finite-size systems the degeneracy at $h=0$ is lifted: the two lowest-energy levels are nondegenerate and their energy difference $\Delta(L)=\Delta(L, h=0)$ vanishes as $L \rightarrow$ $\infty$. The $L$ dependence of $\Delta(L)$ depends on the boundary conditions. For periodic boundary conditions $(\mathrm{PBC})$ and open boundary conditions (OBC) $\Delta(L)$ decreases exponentially with $L, \Delta(L) \sim g^{L}$ [36, 37, while for antiperiodic boundary conditions (ABC) and OFBC [10, 34, it decreases as a power of $L$. Also the finite-size scaling (FSS) behavior close to the transition point is sensitive to the boundary conditions. In particular, the scaling variables may have an exponential or power dependence on $L$.

Studies of the equilibrium behavior for several boundary conditions (PBC, ABC, OBC, EFBC and OFBC) have been reported in Refs. [10, 11, 18. In this work we discuss the out-of-equilibrium dynamic behavior which is observed when a time-dependent longitudinal field $h$ is applied to the system, in the presence of EFBC and OFBC. For this purpose, we consider two limiting protocols [38, 39], that both start from the ground state at an initial value $h_{i}$ of the longitudinal field:

1. At $t=0$ we perform an instantaneous quench of the longitudinal field to a new value $h$ and consider the subsequent unitary evolution. If $h$ is opposite to $h_{i}$, the system effectively crosses the FOQT. Quantum quenches provide the simplest protocol in which a system can be naturally put in out-of-equilibrium conditions - see, e.g., Refs. 40 45;

2. We perform a slow change of the longitudinal field across the FOQT. We consider a linear time dependence

$$
h(t)=-t / t_{s},
$$

where $t_{s}$ is the corresponding time scale. The protocol starts at time $t_{i}=-h_{i} t_{s}$ (we assume $h_{i}>0$ ) so that $h\left(t_{i}\right)=h_{i}$, then the system evolves unitarily, up $t=t_{f}>0$, such that $h\left(t_{f}\right)=h<0$. For $t=0$, the longitudinal field vanishes and the system goes across the FOQT. This protocol is analogous to that implemented for the study of the so-called 
Kibble-Zurek problem, i.e., of the scaling behavior of the amount of defects when a system slowly moves across a continuous quantum transition $46-$ 50.

Different observables are computed during the quantum evolution. In our work we will mostly monitor the local and the average magnetization

$$
m_{x}=\left\langle\Psi(t)\left|\sigma_{x}^{(3)}\right| \Psi(t)\right\rangle, \quad m=\frac{1}{L} \sum_{x=1}^{L} m_{x},
$$

where $|\Psi(t)\rangle$ represents the evolved quantum state at time $t$. In particular, we will consider the normalized quantities

$$
M_{c}(L, h)=\frac{m_{x_{c}}}{m_{0}}, \quad M(L, h)=\frac{m}{m_{0}},
$$

where $x_{c}$ is the central site of the chain, for $L$ odd, or one of the two central sites, for $L$ even. The normalization of $M_{c}$ and $M$ is such that they take the values \pm 1 in the two phases coexisting at the FOQT (i.e., for any fixed, positive or negative, value of the longitudinal field) for any $g<1$, in the limit $L \rightarrow \infty$.

The dynamic behavior at a FOQT has already been discussed for neutral boundary conditions 21, such as $\mathrm{PBC}$ and $\mathrm{OBC}$. Below we show that a significantly different behavior arises when the boundary conditions favor one of the two phases, as in the case of EFBC and OFBC.

\section{EQUILIBRIUM SCALING WITH FIXED BOUNDARY CONDITIONS}

Before addressing the out-of-equilibrium dynamic behavior, we summarize the known results for the equilibrium low-energy properties of the quantum Ising chain with EFBC and OFBC.

\section{A. Quantum Ising chain with EFBC}

Let us first discuss the behavior of the system in the presence of EFBC. Without loss of generality, because of the obvious up-down symmetry, we can fix the spins to the states $|\downarrow\rangle$ at both boundaries, thereby favoring the negative-magnetization phase. For $h=0$, at variance with what happens for neutral boundary conditions, the gap $\Delta(L)$ does not vanish for $L \rightarrow \infty$. Indeed, one has [34]

$$
\Delta(L)=4(1-g)+\frac{5 g \pi^{2}}{(1-g) L^{2}}+O\left(L^{-3}\right),
$$

and $m_{x_{c}} \rightarrow-m_{0}$ for $L \rightarrow \infty$. Since the boundaries favor a negative magnetization, at $h=0$ the system is effectively within the negative-magnetization phase. The transition to the phase with positive magnetization occurs at a positive value of $h$. Indeed, the observables around $h=0$ depend smoothly on $h$, up to a pseudotransition value $h_{t r}(L)$, where the system undergoes a sharp transition to the positively magnetized phase. Such value corresponds to the minimum $\Delta_{m}(L)$ of the gap $\Delta(L, h)$, and, for large $L$, it converges to $h=0$. Its large- $L$ asymptotic behavior is [11]

$$
h_{t r}(L)=\eta(g) L^{-1}+a(g) L^{-5 / 3}+O\left(L^{-2}\right),
$$

where $\eta(g)$ decreases with approaching the continuous transition point $g=1$. The minimum $\Delta_{m}(L)$ behaves exponentially with increasing $L$ :

$$
\Delta_{m}(L) \sim e^{-b(g) L},
$$

where $b(g)$ decreases with approaching $g=1$ [51].

The lowest levels around $h=h_{t r}(L)$ display an avoided-level crossing phenomenon, interpolating the ground states for $h<h_{t r}(L)$ and $h>h_{t r}(L)$. The first one is a negatively magnetized state, while the second one is characterized by a positive local magnetization in the central part of the chain and by two negatively magnetized regions at the boundaries. Note, finally, that in the EFBC case there is an infinite number of states that become degenerate with the ground state for $L \rightarrow \infty$. Indeed, we have [1]

$$
\Delta^{(n)}\left[L, h_{t r}(L)\right] \equiv E_{n}-E_{0}=O\left(L^{-1}\right) \quad \text { for } n \geq 2,
$$

corresponding to the spectrum of kink-antikink states in the presence of an external $O\left(L^{-1}\right)$ magnetic field. This is at variance with the $\mathrm{PBC}$ and $\mathrm{OBC}$ case, where $\Delta^{(n)}(L, 0)$ is finite for $L \rightarrow \infty$ for any $n \geq 2$. Although $\Delta^{(n)}\left[L, h_{t r}(L)\right]$ vanishes for any $n$ in the infinite-volume limit, it is important to stress that the rate is different for $n=1$ (exponential in $L$ ) and for $n \geq 2(1 / L)$.

Around $h_{t r}(L)$, FSS holds. The corresponding scaling variable is the ratio between the energy variation associated with the longitudinal field around $h=h_{t r}(L)$ and the gap $\Delta_{m}(L)$ [11, that is

$$
\kappa_{e}=\frac{2 m_{0}\left[h-h_{t r}(L)\right] L}{\Delta_{m}(L)} .
$$

In the FSS limit at fixed $\kappa_{e}$, the energy gap $\Delta(L, h)$, the average and local central magnetization defined in Eq. (5), with $m_{x}=\left\langle 0_{h}\left|\sigma_{x}^{(3)}\right| 0_{h}\right\rangle\left(\left|0_{h}\right\rangle\right.$ is the ground state at the given $h$ and $L)$, behave as [11]

$$
\begin{aligned}
\Delta(L, h) & \approx \Delta_{m}(L) \mathcal{D}_{E}\left(\kappa_{e}\right), \\
M_{c}(L, h) & \approx \mathcal{M}_{c E}\left(\kappa_{e}\right), \\
M(L, h) & \approx \mathcal{M}_{E}\left(\kappa_{e}\right) .
\end{aligned}
$$

Since the higher excited states decouple from the two lowest levels, $\Delta^{(n)}(L, h) / \Delta(L, h) \sim e^{b(g) L} / L$ for any $n \geq$ 2 , one can compute the scaling functions by considering 
only the two lowest levels. A straightforward calculation gives 11 .

$$
\begin{aligned}
\mathcal{D}_{E}\left(k_{e}\right) & =\mathcal{D}_{2 l}\left(\kappa_{e} / c\right), & \mathcal{D}_{2 l}(x) & =\sqrt{1+x^{2}} \\
\mathcal{M}_{c E}\left(k_{e}\right) & =\mathcal{M}_{2 l}\left(\kappa_{e} / c\right), & \mathcal{M}_{2 l}(x) & =\frac{x}{\sqrt{1+x^{2}}}
\end{aligned}
$$

where $c$ is an appropriate $g$-dependent normalization constant. The asymptotic FSS is approached with exponentially suppressed corrections. It is also possible to compute the scaling function for the average magnetization, but in this case one has to take into account the inhomogeneous behavior at the boundaries [11.

We point out that this is not the end of the story, since another peculiar scaling behavior emerges for $h>h_{t r}(L)$, where the low-energy states are characterized by kinkantikink structures. It is related to the behavior of the domain walls between the spatially separated negatively and positively magnetized regions. Indeed, for $h>h_{t r}(L)$ the central part of the chain is positively magnetized, while close to the boundaries, the local magnetization is negative. As argued in Ref. 11, the size

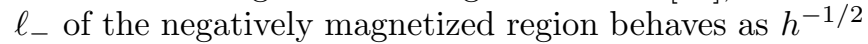
in the large- $L$ limit. Then, the average magnetization is simply $M \approx\left(1-2 \ell_{-} / L\right)-2 \ell_{-} / L=1-4 \ell_{-} / L$. Since $\ell_{-}^{2} / L^{2} \sim 1 / h L^{2}$, we predict $M(L, h) \approx f_{m}(u)$, with $u=h L^{2}$. This scaling behavior holds only for $h>h_{t r}(L)$, i.e., for $u>u_{\min }=h_{t r}(L) L^{2}$. Since $h_{t r}(L) L^{2} \rightarrow \infty$ when $L \rightarrow \infty$, the range of validity of this scaling behavior shrinks as $L$ increases.

\section{B. Quantum Ising chain with OFBC}

OFBC give rise to a spatially dependent local magnetization, whose average $M$ vanishes for $h=0$ by symmetry. For $h=0$ the gap $\Delta(L)$ behaves as $[10,34$ ]

$$
\Delta(L)=\frac{3 g \pi^{2}}{(1-g) L^{2}}-\frac{6 g^{2} \pi^{2}}{(1-g)^{2} L^{3}}+O\left(L^{-4}\right) .
$$

Note that the $L^{-2}$ behavior of the gap differs from the behavior in the presence of $\mathrm{PBC}$ and $\mathrm{OBC}$, where the gap decreases exponentially, $\Delta(L) \sim g^{L}$. This is related to the fact that the low-energy states are one-kink states (for $g \rightarrow 0$ they are combination of states in which there is a single pair of antiparallel spins), which behave as one-particle states with $O\left(L^{-1}\right)$ momenta.

Low-energy properties show FSS, the relevant scaling variable $\kappa_{O}$ being the ratio between the energy associated with magnetic perturbation, $E_{h}(L) \approx 2 m_{0} L h$, and the gap $\Delta(L)$ at $h=0$ [10],

$$
\kappa_{o}=\frac{2 m_{0} L h}{\Delta(L)} \sim h L^{3} .
$$

The FSS limit corresponds to $L \rightarrow \infty$ and $h \rightarrow 0$, keeping $\kappa_{o}$ fixed. In this limit, the energy gap and the rescaled magnetization associated with the ground state behave as

$$
\begin{aligned}
\Delta(L, h) & \approx \Delta(L) \mathcal{D}_{O}\left(\kappa_{o}\right), \\
M(L, h) & \approx \mathcal{M}_{O}\left(\kappa_{o}\right),
\end{aligned}
$$

where $\mathcal{D}_{O}$ and $\mathcal{M}_{O}$ are universal functions independent of $g$. The above equilibrium FSS predictions have been numerically confirmed in Ref. [10]. Corrections to the asymptotic FSS behavior scale as $1 / L$.

\section{DYNAMIC SCALING WITH EFBC}

As shown in Ref. [11, systems with neutral boundary conditions, such as PBC and OBC, develop a dynamic scaling behavior at a FOQT when an instantaneous quench is performed. The corresponding scaling variables are the equilibrium variable $\kappa=2 m_{0} h L / \Delta(L)$ and $\theta=t \Delta(L)$, where $t$ is the time. We expect a similar scaling behavior in the case of EFBC, provided one takes into account that in a finite-size system the transition effectively occurs at $h \approx h_{t r}(L)$, see Eq. (7). In the following we will discuss and verify the dynamic scaling theory when an instantaneous quench is performed. We will then extend these results to the case in which the longitudinal field is slowly varied across the transition.

\section{A. Instantaneous quenches of $h$}

We consider an instantaneous quench at $t=0$, from a longitudinal field $h_{i}$ to a new field $h$. For EFBC, the effective transition occurs at $h_{t r}(L)$, so that we choose $h_{i}>h_{t r}(L)$ and $h<h_{t r}(L)$, in order to observe the dynamic behavior across the transition. As discussed in Ref. [11, the dynamic scaling depends on the equilibrium FSS variable computed at the initial and final value of the applied external field. For EFBC, we therefore consider $\kappa_{e}$ and $\kappa_{e i}$, corresponding to the final and initial longitudinal fields $h$ and $h_{i}$, respectively. As for the scaling variable associated with the time $t$, we take into account that the relevant energy scale is the gap $\Delta_{m}(L)$ at the pseudo-transition point $h_{t r}(L)$, so that we consider

$$
\theta_{e}=t \Delta_{m}(L)
$$

In the FSS limit $L \rightarrow \infty, h_{i}, h \rightarrow 0, t \rightarrow \infty$, keeping $\kappa_{e i}, \kappa_{e}$, and $\theta_{e}$ fixed, the local central magnetization, defined in Eq. (5), has the asymptotic behavior

$$
M_{c}\left(L, h_{i}, h, t\right) \approx \mathcal{Q}_{c E}\left(\kappa_{e i}, \kappa_{e}, \theta_{e}\right) .
$$

The average magnetization $M$ should behave analogously.

As in the case of neutral boundary conditions, since the higher excited states decouple from the two lowest-energy levels, the dynamic scaling functions can be computed using a two-level truncation of the spectrum [10, 11, 22. 
One only considers the two-dimensional reduced Hilbert subspace generated by $|-\rangle$ and $|+\rangle$, which are the ground states for $h<h_{t r}(L)$ and $h>h_{t r}(L)$, respectively. The effective evolution in this subspace is determined by the Schrödinger equation

$$
i \partial_{t}\left|\psi_{r}(t)\right\rangle=H_{r}(t)\left|\psi_{r}(t)\right\rangle
$$

where the effective Hamiltonian $H_{r}(t)$ reads [10, 21]

$$
H_{r}=m_{0} h L \sigma^{(3)}+\frac{1}{2} \Delta_{m} \sigma^{(1)} .
$$

In order to determine the scaling function $\mathcal{Q}_{c E}$, one needs to compute the expectation value $\left\langle\Psi(t)\left|\sigma^{(3)}\right| \Psi(t)\right\rangle$, where $|\Psi(t)\rangle$ is the state obtained starting from the ground state for the Hamiltonian with field $h_{i}$. A straightforward calculation gives

$$
\begin{aligned}
\mathcal{Q}_{c E, r}\left(\kappa_{e i}, \kappa_{e}, \theta_{e}\right) & =\cos \left(\alpha-\alpha_{i}\right) \cos \alpha \\
& +\cos \left(\theta_{e} \sqrt{1+\kappa_{e}^{2}}\right) \sin \left(\alpha-\alpha_{i}\right) \sin \alpha,
\end{aligned}
$$

where $\tan \alpha=\kappa_{e}^{-1}$ and $\tan \alpha_{i}=\kappa_{e i}^{-1}$. One can thus predict the scaling function appearing in Eq. (17),

$$
\mathcal{Q}_{c E}\left(\kappa_{e i}, \kappa_{e}, \theta_{e}\right)=\mathcal{Q}_{c E, r}\left(\kappa_{e i} / c_{1}, \kappa_{e} / c_{2}, \theta_{e} / c_{3}\right),
$$

where $c_{1}, c_{2}$, and $c_{3}$ are three nonuniversal modeldependent constants.

To verify the scaling prediction (17), in Fig. 1 we report numerical results for $g=0.5, \kappa_{e i}=1$ and $\kappa_{e}=-1$ [52]. Note that it is first required to determine $h_{t r}(L)$ and the corresponding gap $\Delta_{m}(L)$, which enter the definition of the above rescaled quantities [51]. In the upper panel we plot the normalized central magnetization (5) as a function of the rescaled time 16 for several values of $L$. The data for $L=14$ and $L=12$ fall on top of each other, confirming the validity of the scaling Ansatz. As is clearly visible, the curves display Rabi oscillations, which naturally emerge from the dynamics of a two-level system [21]. The continuous black line on top of the colored ones is a fit $\left(c_{2}\right.$ and $c_{3}$ are the fit parameters, while $c_{1}$ is obtained by matching the value of the magnetization for $t=0$ ) of the numerical data at the largest available size $(L=14)$ to the two-level prediction 21$)$. The agreement with the numerical data is excellent, confirming the two-level description of the dynamics.

The lower panel focuses on the finite-size approach to the asymptotic behavior, which is consistent with an exponential behavior of the type $M_{c}(L) \sim a+b e^{-c L}$, both for the pre-quench equilibrium state $\left(\theta_{e}=0\right)$ and also along the post-quench dynamics $\left(\theta_{e}>0\right)$. We limited our analysis to $L=12$, because it was impossible to reach a degree of accuracy in the temporal evolution sufficient to observe a clear exponential decay at larger $L$.

We simulated the post-quench dynamics of the quantum Ising chain with EFBC for several other values of the transverse field $g$ and rescaled fields $\kappa_{e i}, \kappa_{e}$, always obtaining a neat consistency with the effective two-level prediction presented above. In the remainder of our work, we will thus assume its validity for any type of dynamic behavior in the appropriate FSS limit.
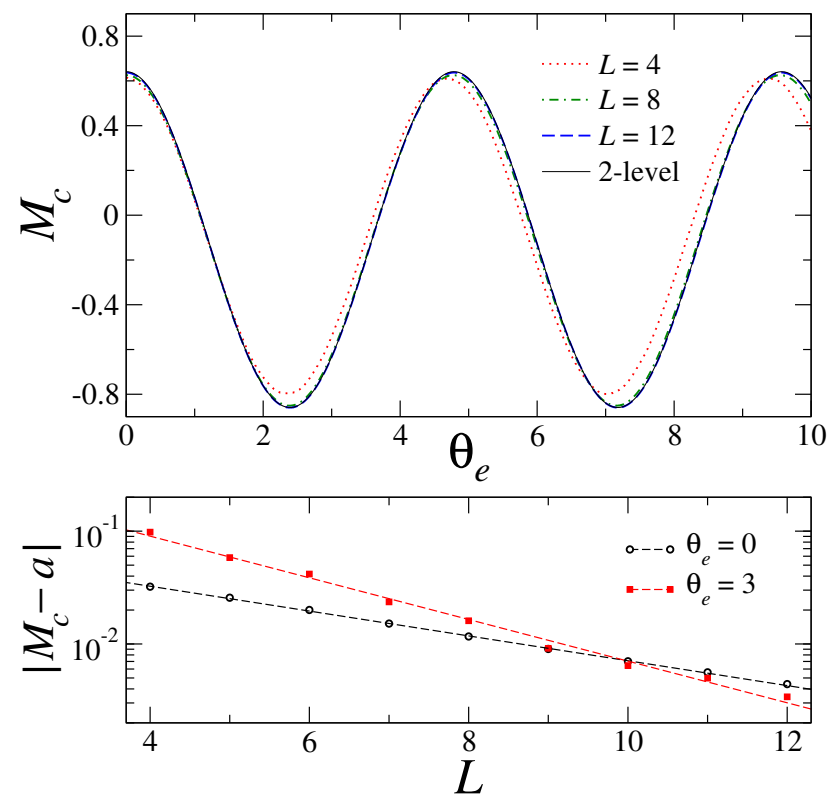

FIG. 1: Upper panel: normalized local magnetization $M_{c}$ as a function of the rescaled time variable $\theta_{e}$, after a sudden quench of the longitudinal field. We consider EFBC and fix $g=0.5, \kappa_{e i}=+1, \kappa_{e}=-1$. Different colored data sets correspond to different chain lengths $L$. The continuous black line represents a fit of the numerical data for $L=14$ (not shown in the figure, as they are barely distinguishable from those at $L=12$ ) to Eq. 21. Lower panel: Difference between the numerically computed $M_{c}$ and the asymptotic value, as a function of $L$. The dashed lines correspond to fits to $M_{c}(L) \sim$ $a+b e^{-c L}$. Black circles stand for the static case $\theta_{e}=0$, red squares are for $\theta_{e}=3$.

\section{B. Slow variations of $h$}

We now discuss a second protocol, in which $h$ varies slowly across the FOQT, generalizing the theory discussed in Ref. 22. We assume that $h$ varies as $h(t)=$ $-t / t_{s}$, and that the dynamics starts from the ground state at a finite $h_{i}>h_{t r}(L)$ and ends at $h_{f}<h_{t r}(L)$. It is convenient to introduce a new time variable

$$
\hat{t} \equiv t+t_{s} h_{t r}(L)
$$

such that $\hat{t}=0$ corresponds to the pseudo-transition point. The natural scaling variables are the equilibrium scaling variable $\kappa_{e}$ defined in Eq. 10 , with $h$ replaced by $h(t)$, that is

$$
\omega_{e}=-\frac{2 m_{0} L}{\Delta_{m}(L)} \frac{\hat{t}}{t_{s}},
$$

and

$$
\hat{\theta}_{e} \equiv \hat{t} \Delta_{m}(L)
$$

It is also convenient to define a related scaling variable

$$
v_{e}=-\hat{\theta}_{e} / \omega_{e}=\frac{t_{s} \Delta_{m}(L)^{2}}{2 m_{0} L},
$$


which is independent of $t$. The dynamic scaling limit is obtained by taking $\hat{t}, t_{s}, L \rightarrow \infty$, keeping the scaling variables $v_{e}$ and $\omega_{e}$ or $\hat{\theta}_{e}$ fixed. In this limit, the local central magnetization is expected to obey the asymptotic FSS behavior

$$
M_{c}\left(L, t_{s}, t\right) \approx \mathcal{S}_{c E}\left(v_{e}, \omega_{e}\right)=\hat{\mathcal{S}}_{c E}\left(v_{e}, \hat{\theta}_{e}\right) ;
$$

an analogous relation holds for the average magnetization $M$. In the adiabatic limit $\left(t, t_{s} \rightarrow \infty\right.$ at fixed size $)$, the equilibrium FSS must be recovered, so that

$$
\mathcal{S}_{c E}\left(v_{e} \rightarrow \infty, \omega_{e}\right)=\mathcal{M}_{c E}\left(\omega_{e}\right),
$$

with $\mathcal{M}_{c E}$ given by Eq. $12 \mathrm{~b}$.

In the FSS limit we can perform a two-level truncation of the spectrum to compute the scaling functions (as before, the two levels are indicated as $|-\rangle$ and $|+\rangle$ ). Starting from Eq. 19 , we obtain the effective time-dependent Hamiltonian

$$
H_{r}(t)=-\frac{m_{0} \hat{t} L}{t_{s}} \sigma^{(3)}+\frac{1}{2} \Delta_{m} \sigma^{(1)} .
$$

It is immediate to recognize that this Hamiltonian is analogous to the one that appears in the Landau-Zener problem 53. If $\psi_{r}(t)$ is the solution of Schrödinger equation with the initial condition $\psi_{r}\left(t_{i}\right)=|+\rangle(|+\rangle$ is the positive eigenvalue of $\sigma^{(3)}$ ), using the results of Ref. [54] for the Landau-Zener problem, we obtain

$$
\left|\psi_{r}(t)\right\rangle=C_{-}\left(v_{e}, \omega_{e}\right)|-\rangle+C_{+}\left(v_{e}, \omega_{e}\right)|+\rangle,
$$

where $C_{ \pm}$are known functions of the scaling variables $v_{e}$ and $\omega_{e}$. The dynamic scaling of the local central magnetization can be computed by taking the ground-state expectation value of $\sigma^{(3)}$. This allows us to compute the dynamic FSS function $\mathcal{S}_{c E}$ defined in Eq. (26) apart from a rescaling of the scaling variables. For the two-level system we obtain

$$
\begin{aligned}
\mathcal{S}_{c E, r}\left(v_{e}, \omega_{e}\right) & =\left\langle\psi_{r}(t)\left|\sigma^{(3)}\right| \psi_{r}(t)\right\rangle \\
& =\left|C_{+}\left(v_{e}, \omega_{e}\right)\right|^{2}-\left|C_{-}\left(v_{e}, \omega_{e}\right)\right|^{2} \\
& =1-\frac{1}{4} v_{e} e^{-\frac{\pi v_{e}}{16}}\left|D_{-1+i \frac{v_{e}}{8}}\left(e^{i \frac{3 \pi}{4}} \sqrt{2 v_{e}} \omega_{e}\right)\right|^{2},
\end{aligned}
$$

where $D_{\nu}(z)$ is the parabolic cylinder function [55]. The scaling function $\mathcal{S}_{c E}$ can be related to $\mathcal{S}_{c E, r}$ by simply rescaling the arguments by constant nonuniversal factors, as already discussed for $\mathcal{Q}_{c E}$, see Eq. (21).

\section{DYNAMIC SCALING WITH OFBC}

In this section we focus on the dynamic behavior of the quantum Ising chains with OFBC. As we shall observe, the dynamic features of the out-of-equilibrium behavior close to the FOQT are characterized by time scales that increase as powers of the size, at variance with neutral boundary conditions and $\mathrm{EFBC}$, where the time scale increases exponentially with $L$. In this case, it is not possible to exploit a two-level truncation of the spectrum in order to determine the asymptotic FSS behavior.

\section{A. Instantaneous quenches of $h$}

We first consider the dynamic behavior arising from an instantaneous quench of the external longitudinal field from $h_{i}$ to $h$. Dynamic scaling depends on the equilibrium scaling variable $\kappa_{o}$ defined in Eq. (14), computed for the initial and final values of the field. Therefore, we introduce $\kappa_{o i}$ corresponding to the initial field $h_{i}$ and $\kappa_{o}$ which corresponds to the post-quench field $h$. Moreover, we introduce a scaling variable associated with the time $t$,

$$
\theta_{o}=t \Delta(L),
$$

where $\Delta(L)$ is the gap at $h=0$. Note that $\Delta(L)$ scales as a power of $L$, see Eq. (13), so that $\theta_{o} \sim t L^{-2}$. We can then define a dynamic FSS limit $L \rightarrow \infty, h_{i}, h \rightarrow 0$, $t \rightarrow \infty$, keeping $\kappa_{o i}, \kappa_{o}$, and $\theta_{o}$ fixed. In this limit we expect

$$
M\left(L, h_{i}, h, t\right) \approx \mathcal{Q}_{O}\left(\kappa_{o i}, \kappa_{o}, \theta_{o}\right),
$$

and an analogous relation for the local central magnetization. Scaling corrections are expected to behave as $1 / L$. The scaling function defined in Eq. (32) should be universal, apart from possible multiplicative normalization of the scaling variables. In particular, the same behavior, but with different normalization constants, is expected for different values of the Hamiltonian parameter $g$.

The dynamic FSS behavior, Eq. (32), is supported by the results of our numerical simulations. In Fig. 2 we show the average magnetization as a function of $\theta_{o}$, for fixed values of $\kappa_{o i}$ and $\kappa_{o}$ : in the upper panel we fix $h_{i}$ and $h$ at opposite sides of the transition point $h=0$, while in the lower panel $h_{i}$ and $h$ have the same sign so that the system is not going across the FOQT. In both cases, as $L$ increases, the data nicely approach an asymptotic function. They show oscillations in time, which, however, are not sinusoidal as observed with EFBC (see Fig. 11. This is related to the fact that the dynamics for OFBC cannot be schematized in terms of a two-level dynamics, due to the presence of a tower of excited states, such that their energy differences $\Delta^{(n)}=E_{n}-E_{0}$ decrease with the same power of $L$ for any $n \geq 1$.

As expected, the convergence to the scaling behavior is characterized by $1 / L$ corrections. This has been explicitly verified in our numerics: the magnetization data at fixed $\theta_{o}$, plotted in Fig. 3, scale linearly as a function of $L^{-1}$ as soon as $L \gtrsim 10$. We have reported only the results for $\kappa_{o i}=+1, \kappa_{o}=-1$, but qualitatively analogous results have been obtained for other values of $\theta_{o}$ and also for different $\kappa_{o i}$ and $\kappa_{o}$. It is interesting to note that the extrapolated asymptotic value for $L \rightarrow \infty$ for fixed scaling variables does not depend on the specific choice of $g$, within the numerical accuracy. Apparently, the $g$-dependences of $m_{0}$ and of the amplitude of the gap $\Delta(L)$, entering the definitions of the scaling variables, provide the correct normalizations, without the need of further $g$-dependent rescalings. One can reach the same 


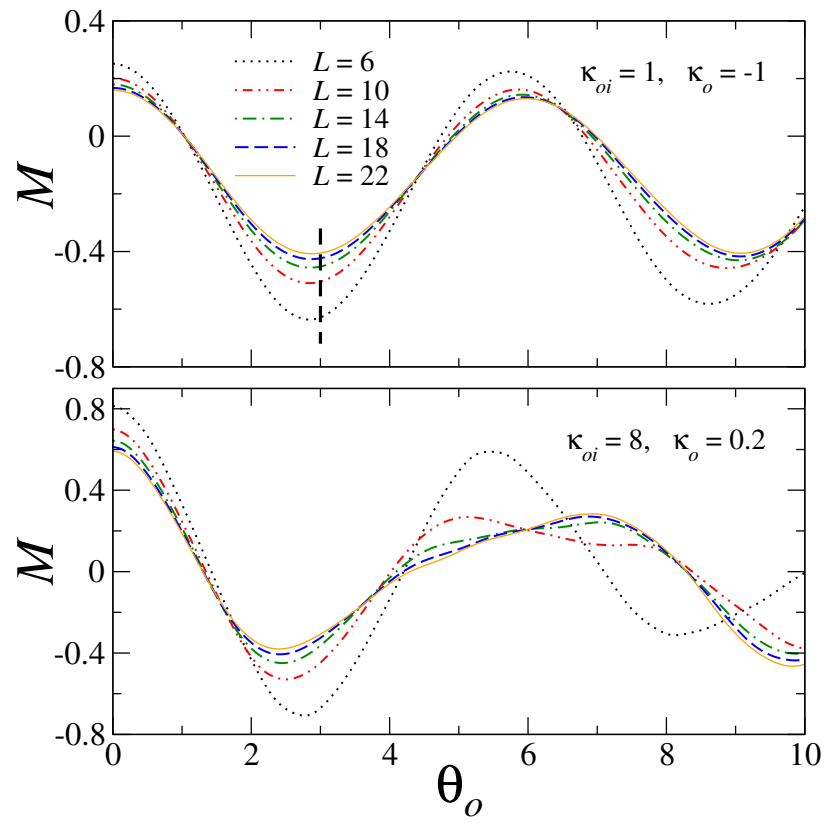

FIG. 2: Average magnetization $M$ for the quantum Ising chain with OFBC, after a sudden quench of the longitudinal field close to the FOQT, as a function of the rescaled time variable $\theta_{o}$. We fix $g=0.5$ and the rescaled variables $\kappa_{o i}$ and $\kappa_{0}$ (in the upper panel $\kappa_{o i}=-\kappa_{o}=1$, while in the lower panel $\kappa_{o i}=8, \kappa_{o}=0.2$ ). Different data sets are for various chain lengths $L$, as indicated in the legend.

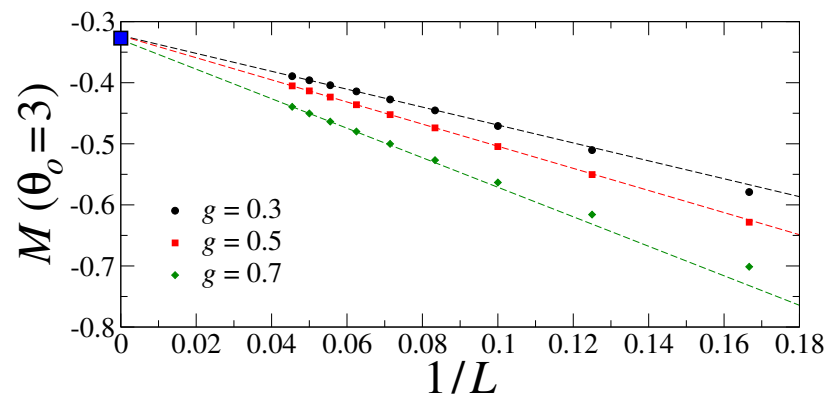

FIG. 3: Average magnetization as a function of $1 / L$ for $\theta_{o}=3, \kappa_{o i}=+1$, and $\kappa_{o}=-1$ (dashed vertical line in the upper panel of Fig. 2). Different symbols denote the numerical results for three values of the transverse field $g$ (see legend). They confirm that scaling corrections are $O\left(L^{-1}\right)$, as shown by the dashed lines, which are $1 / L$ fits of four data corresponding to the largest available sizes $(L=16,18,20$, and $22)$ to $M \sim M_{\infty}+a / L$. The asymptotic values $M_{\infty}$ appear to be approximately independent of $g$ within our numerical precision: $M_{\infty} \approx 0.323,0.323,0.330$, for $g=0.3,0.5,0.7$, respectively.

conclusions by analyzing other observables, as the central magnetization $M_{c}$ (not shown). Note also that, although for OFBC the corrections to the asymptotic FSS behavior decay only as a power of $L$, systems of length $L \leq 22$ were sufficient to observe the convergence to the asymptotic behavior.

\section{B. Slow variations of $h$}

We finally discuss and analyze the protocol, in which the longitudinal field varies as in Eq. (3). We start from the ground state at a finite $h_{i}>0$ and stop at $h_{f}<0$, thus crossing the FOQT located at $h=0$. As we already discussed in Sec. IVB the scaling variables are $\theta_{o}$ and

$$
\omega_{o}=-\frac{2 m_{0} L}{\Delta(L)} \frac{t}{t_{s}},
$$

obtained by replacing $h$ with $h(t)$ in the definition of the equilibrium scaling variable $\kappa_{o}$, defined in Eq. (14). It is also convenient to define the time-independent scaling variable

$$
v_{o} \equiv\left|\theta_{o} / \omega_{o}\right|=\frac{\Delta(L)^{2} t_{s}}{2 m_{0} L},
$$

and the (asymptotically) size-independent scaling variable

$$
\tau_{o} \equiv \operatorname{sign}(t)\left|\omega_{o}\right|^{2 / 5}\left|\theta_{o}\right|^{3 / 5} .
$$

The dynamic FSS limit is obtained by taking $L \rightarrow \infty$, $t_{s} \rightarrow \infty$, and $t \rightarrow \infty$, at $v_{o}$ and $\theta_{o}$ (or any other pair of scaling variables) fixed. In this limit, since $\Delta(L) \sim L^{-2}$, see Eq. (16), the scaling variables scale as

$$
\omega_{o} \sim-\left(t / t_{s}\right) L^{3}, \quad v_{o} \sim t_{s} L^{-5}, \quad \tau_{o} \sim t / t_{s}^{2 / 5} .
$$

Note that these scaling variables can also be derived using the fact that the relevant low-energy configurations are made of kinks and antikinks. A kink in the presence of an external magnetic field can be effectively described by a particle subject to a linear potential. Indeed, if the kink is located at a distance $x$ from the center of the chain, the magnetic field $h$ induces a linear potential $H_{h}=-2 h x$. Correspondingly, the energy spacing of the low-energy levels is $\delta E_{n}=O\left(h^{2 / 3}\right)$ [1], 56 58. Therefore, we can consider the scaling variables

$$
\frac{|h(t)|^{2 / 3}}{\Delta(L)} \sim \omega_{o}^{3 / 2} \quad t|h(t)|^{2 / 3} \sim \tau_{o}^{3 / 5} .
$$

In the dynamic finite-size scaling limit, keeping the starting longitudinal field $h_{i}$ fixed (it is not rescaled with $L$ ), we expect the average magnetization to behave as

$$
M\left(L, h_{i}, t_{s}, t\right) \approx \mathcal{S}_{O}\left(v_{o}, \theta_{o}\right) .
$$

The scaling function does not depend on $h_{i}$. This is due to the fact that, for finite $h>0$, the gap is finite in the limit $L \rightarrow \infty$. Therefore, for $t_{s} \rightarrow \infty$, the dynamics is always adiabatic and the system goes through the instantaneous ground states as long as $h>0$. An outof-equilibrium behavior occurs only in an interval around $h=0$ that shrinks as $L^{-3}$, or equivalently $t_{s}^{-3 / 5}$, since $\omega_{o} \sim h(t) L^{3}$ or $\tau_{o} \sim h(t) t_{s}^{3 / 5}$ are kept fixed in the dynamic FSS limit, see Eq. 36. The dynamic scaling 


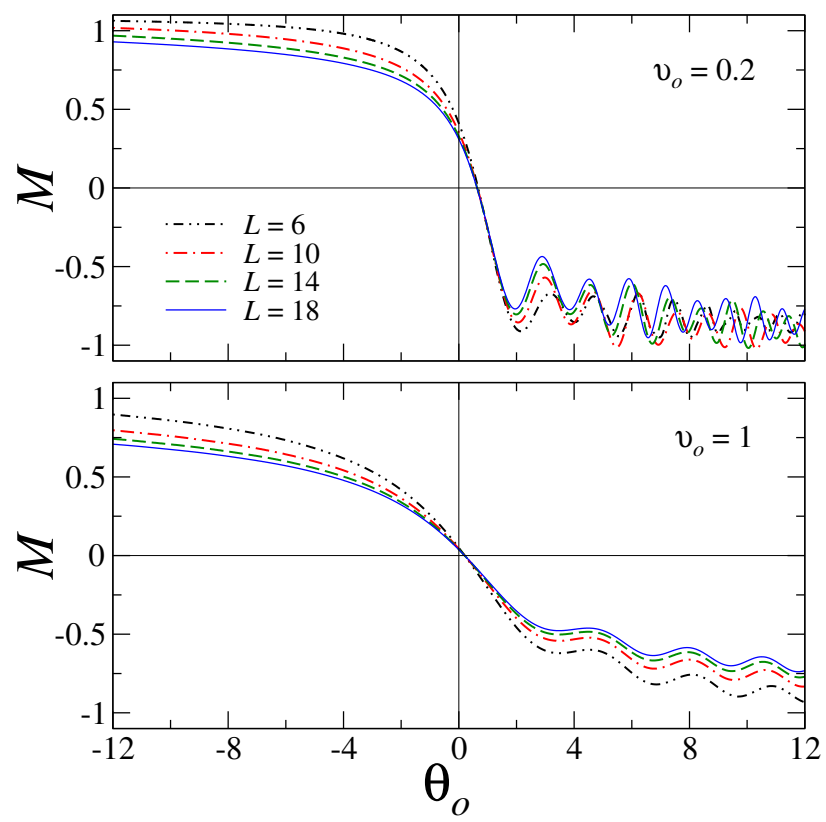

FIG. 4: Average magnetization with OFBC as a function of the rescaled time variable $\theta_{o}$ for different values of $L$. The longitudinal field varies according to Eq. (3) and $h_{i}=1$. This starting value has been chosen sufficiently far from the transition point, so as to ensure that all the reported curves are unaffected by the choice, on the scale we are interested in. All data sets have been obtained for $g=0.7$. In the upper panel $v_{0}=0.2$, in the lower panel $v_{0}=1$.

behavior around $h=0$ is thus not expected to depend on the choice of the initial $h_{i}>0$. For the same reason also the final value $h_{f}$ is irrelevant for the scaling behavior. It is easy to realize that the scaling behavior (38) also holds for generic time-dependent $h(t)$. Indeed, if $h(t)=a t+O\left(t^{2}\right)$, the same scaling behavior is obtained provided we identify $|a|$ with $1 / t_{s}$. The higherorder terms give only rise to scaling corrections. If the linear term is missing $(a=0)$, the appropriate dynamic FSS variables can be straightforwardly obtained by simply considering the leading nonvanishing term in the expansion of $h(t)$.

The equilibrium FSS must be recovered in the adiabatic limit $t, t_{s} \rightarrow \infty$ at fixed $L$ and $t / t_{s}$, thus for $v_{o} \rightarrow \infty$ keeping $\omega_{o}$ fixed. Therefore, we should have

$$
\mathcal{S}_{O}\left(v_{o} \rightarrow \infty, \omega_{o}\right)=\mathcal{M}_{O}\left(\omega_{o}\right)
$$

where $\mathcal{M}_{O}$ enters the equilibrium FSS relation $15 \mathrm{~b}$.

Numerical results for the average magnetization $M$ as a function of the rescaled time $\theta_{o}$ are reported in Fig. 4. for two different values of the scaling variable $v_{o}$. We started from the initial field $h_{i}=1$, sufficiently far from the FOQT point $\left(\theta_{0}=h=0\right)$. Unfortunately, we were not able to consider sizes larger than $L=20$, due to the fast increase of the time scale $t_{s}$ with the size, $t_{s} \sim L^{5}$, see Eq. (36). Nevertheless, the data support the FSS behavior predicted in Eq. (38) since, for increasing system size
$L$, the different curves approach an asymptotic function. Note the appearance of wiggles for $\theta_{o}>0$, especially in the upper panel $\left(v_{o}=0.2\right)$, due to the loss of adiabaticity occurring in proximity to the FOQT. Such wiggles are suppressed when $v_{o}$ is increased (bottom panel), i.e. moving towards the adiabatic limit, for which the magnetization becomes an odd function of the rescaled time, $M\left(\theta_{o}\right)=-M\left(-\theta_{o}\right)$. Here we have shown results for a specific choice for the transverse field $g$, but analogous results were obtained for other values of $g<1$, supporting the expected universality with respect to variations of $g$.

\section{CONCLUSIONS}

We have addressed the dynamic behavior of manybody systems at FOQTs, when a Hamiltonian parameter is driven across its FOQT value. Emphasis has been put on systems subject to boundary conditions that favor one of the two phases separated by the FOQT, extending earlier analyses for systems with neutral boundary conditions [21], such as periodic boundary conditions.

We have focused on the paradigmatic quantum Ising chain, whose phase diagram presents a FOQT line, where the transitions are driven by the external longitudinal field $h$. We have studied the out-of-equilibrium dynamic behavior when $h$ is varied across the FOQT for systems with fixed boundary conditions favoring one of the magnetized phases. We have considered equal fixed boundary conditions (EFBC), that both favor the same phase, and opposite fixed boundary conditions (OFBC), that favor different magnetized phases close to the endpoints of the chain. Our results extend previous studies of the equilibrium properties at FOQT for different the boundary conditions, see, e.g., Refs. [10, 11, 17, 18, 35, to the out-of-equilibrium case. It emerges that $\mathrm{EFBC}$ and OFBC lead to remarkable, even qualitatively, differences with respect to the generally considered case of neutral boundary conditions.

We address two different dynamics: an instantaneous quench of the longitudinal field and a protocol in which $h$ varies slowly across the FOQT. As it occurs for neutral boundary conditions 21, one can observe a dynamic finite-size scaling for both EFBC and OFBC. One of the relevant scaling variables is the ratio $\kappa$, that controls the equilibrium finite-size scaling. It is defined as the energy contribution due to $h$ (normalized so that it vanishes at the transition point) and the gap at the transition, see Eqs. 10 and 140 for EFBC and OFBC, respectively. Note that, in the EFBC case, for finite values of $L$, one should consider the pseudo-transition point $h_{t r}(L) \sim L^{-1}$, where the gap is minimal. We also introduce a a second scaling variable related to the time. As we are considering a unitary dynamics, it is natural to choose $\theta=t \Delta$, see Eqs. (16) and (31) for the two boundary conditions, respectively. The emerging dynamic FSS is characterized by very different time scales. The time 
scale of the dynamic behavior across the FOQT increases exponentially with the size $L$ for EFBC, while it increases as a power of the size for OFBC. This is essentially related to the fact that the minimum gap decreases exponentially with $L$ in the case of EFBC and as a power, $\Delta \sim L^{-2}$, for OFBC.

We believe that the general dynamic scenario emerging in the quantum Ising chain along its FOQT line and, in particular, the dependence on the boundary conditions, is quite general. The general ideas should apply to other systems, also in higher dimensions. For example, higher-dimensional quantum Ising models present similar phase diagrams, with a FOQT line where transitions are driven by the longitudinal field $h$, ending at a continuous quantum transition. They are expected to display similar behaviors along the FOQT line, when subject to neutral boundary conditions, or fixed boundary conditions favoring one of the two magnetized phase. In a sense, the dramatic sensitivity of the equilibrium and dynamic properties on the boundary conditions should be considered as a broad feature of FOQTs, distinguishing them from their continuous counterparts. Indeed, the large spectrum of behaviors present at FOQTs, with time scales that increase either exponentially or as a power of the size, is not observed at continuous transitions, where only power laws are typically observed. The strong dependence of the dynamics on the boundary conditions has been also reported at classical first-order transitionssee, e.g., Refs. 23 33 .

Finally we mention that the dynamic scaling behaviors discussed here may be observed in relatively small systems. Therefore, given the need for high accuracy without necessarily reaching scalability to large sizes, we believe that the available technology for probing the coherent quantum dynamics of interacting systems, such as with ultracold atoms in optical lattices 59 61, trapped ions 62 69, as well as Rydberg atoms in arrays of optical microtraps [70, 71], could offer possible playgrounds where the behaviors we envisioned at FOQTs can be observed.
[1] S. L. Sondhi, S. M. Girvin, J. P. Carini, and D. Shahar, Continuous quantum phase transitions, Rev. Mod. Phys. 69, 315 (1997).

[2] S. Sachdev, Quantum Phase Transitions, (Cambridge Univ. Press, 1999).

[3] M. Vojta, Quantum phase transitions, Rep. Prog. Phys. 66, 2069 (2003).

[4] M. N. Barber, Finite-size scaling, in Phase transitions and critical phenomena, vol. 8, page 145, C. Domb and J. L. Lebowitz eds. (Academic Press, London 1983).

[5] Finite Size Scaling and Numerical Simulations of Statistical Systems, ed. V. Privman (World Scientific, 1990).

[6] A. Pelissetto and E. Vicari, Critical Phenomena and Renormalization Group Theory, Phys. Rep. 368, 549 (2002).

[7] F. M. Gasparini, M. O. Kimball, K. P. Mooney, and M. Diaz-Avilla, Finite-size scaling of ${ }^{4} \mathrm{He}$ at the superfluid transition, Rev. Mod. Phys. 80, 1009 (2008).

[8] M. Campostrini, A. Pelissetto, and E. Vicari, Finite-size scaling at quantum transitions, Phys. Rev. B 89, 094516 (2014).

[9] K. Binder, Theory of first-order phase transitions, Rep. Prog. Phys. 50, 783 (1987).

[10] M. Campostrini, J. Nespolo, A. Pelissetto, and E. Vicari, Finite-size scaling at first-order quantum transitions, Phys. Rev. Lett. 113, 070402 (2014).

[11] A. Pelissetto, D. Rossini, and E. Vicari, Finite-size scaling at first-order quantum transitions when boundary conditions favor one of the two phases, Phys. Rev. E 98, 032124 (2018).

[12] V. Piazza, V. Pellegrini, F. Beltram, W. Wegscheider, T. Jungwirth, and A. H. MacDonald, First-order phase transitions in a quantum Hall ferromagnet, Nature 402, 638 (1999).

[13] T. Vojta, D. Belitz, T. R. Kirkpatrick, and R. Narayanan, Quantum critical behavior of itinerant ferromagnets, Ann. Phys. (Leipzig) 8, 593 (1999).
[14] M. Uhlarz, C. Pfleiderer, and S. M. Hayden, Quantum phase transitions in the itinerant ferromagnet $\mathrm{ZrZn}_{2}$, Phys. Rev. Lett. 93, 256404 (2004).

[15] C. Pfleiderer, Why first order quantum phase transitions are interesting, J. Phys.: Cond. Matter 17, S987 (2005).

[16] W. Knafo, S. Raymond, P. Lejay, and J. Flouquet, Antiferromagnetic criticality at a heavy-fermion quantum phase transition, Nat. Phys. 5, 753 (2009).

[17] M. Campostrini, J. Nespolo, A. Pelissetto, and E. Vicari, Finite-size scaling at first-order quantum transitions of quantum Potts chains, Phys. Rev. E 91, 052103 (2015).

[18] M. Campostrini, A. Pelissetto, and E. Vicari, Quantum transitions driven by one-bond defects in quantum Ising rings, Phys. Rev. E 91, 042123 (2015).

[19] A. Yuste, C. Cartwright, G. De Chiara, and A. Sanpera, Entanglement scaling at first order quantum phase transitions, New J. Phys. 20, 043006 (2018).

[20] D. Rossini and E. Vicari, Ground-state fidelity at firstorder quantum transitions, Phys. Rev. E 98, 062137 (2018).

[21] A. Pelissetto, D. Rossini, and E. Vicari, Dynamic finitesize scaling after a quench at quantum transitions, Phys. Rev. E 97, 052148 (2018).

[22] A. Pelissetto, D. Rossini, and E. Vicari, Out-ofequilibrium dynamics driven by localized time-dependent perturbations at quantum phase transitions, Phys. Rev. B 97, 094414 (2018).

[23] B. Nienhuis and M. Nauenberg, First-Order Phase Transitions in Renormalization-Group Theory, Phys. Rev. Lett. 35, 477 (1975).

[24] M. E. Fisher and A. N. Berker, Scaling for first-order phase transitions in thermodynamic and finite systems, Phys. Rev. B 26, 2507 (1982).

[25] V. Privman and M. E. Fisher, Finite-size effects at firstorder transitions, J. Stat. Phys. 33, 385 (1983).

[26] M. E. Fisher and V. Privman, First-order transitions breaking $\mathrm{O}(n)$ symmetry: Finite-size scaling, Phys. Rev. 
B 32, 447 (1985).

[27] M. S. S. Challa, D. P. Landau, and K. Binder, Finitesize effects at temperature-driven first-order transitions, Phys. Rev. B 34, 1841 (1986).

[28] C. Borgs and R. Kotecky, A rigorous theory of finite-size scaling at first-order phase transitions, J. Stat. Phys. 61, 79 (1990).

[29] H. Panagopoulos and E. Vicari, Off-equilibrium scaling across a first-order transition, Phys. Rev. E 92, 062107 (2015).

[30] A. Pelissetto and E. Vicari, Dynamic off-equilibrium transition in systems slowly driven across thermal firstorder transitions, Phys. Rev. Lett. 118, 030602 (2017).

[31] A. Pelissetto and E. Vicari, Dynamic finite-size scaling at first-order transitions, Phys. Rev. E 96, 012125 (2017).

[32] H. Panagopoulos, A. Pelissetto, and E. Vicari, Anomalous finite-size scaling at the thermal first-order transition of systems with disordered boundary conditions, Phys. Rev. D 98, 074507 (2018).

[33] P. Fontana, Scaling behavior of Ising systems at firstorder transitions, J. Stat. Mech. (2019) 063206.

[34] M. Campostrini, A. Pelissetto, and E. Vicari, Quantum Ising chains with boundary terms, J. Stat. Mech. (2015) P11015.

[35] C. R. Laumann, R. Moessner, A. Scardicchio, and S. L. Sondhi, Quantum adiabatic algorithm and scaling of gaps at first-order quantum phase transitions, Phys. Rev. Lett. 109, 030502 (2012).

[36] P. Pfeuty, The one-dimensional Ising model with a transverse field, Ann. Phys. 57, 79 (1970).

[37] G. G. Cabrera and R. Jullien, Role of boundary conditions in the finite-size Ising model, Phys. Rev. B 35, 7062 (1987).

[38] J. Dziarmaga, Dynamics of a quantum phase transition and relaxation to a steady state, Adv. Phys. 591063 (2010).

[39] A. Polkovnikov, K. Sengupta, A. Silva, and M. Vengalattore, Colloquium: Nonequilibrium dynamics of closed interacting quantum systems, Rev. Mod. Phys. 83, 863 (2011).

[40] M. Greiner, O. Mandel, T. Esslinger, T. W. Hänsch, and I. Bloch, Quantum phase transition from a superfluid to a Mott insulator in a gas of ultracold atoms, Nature 415, 39 (2002).

[41] T. Kinoshita, T. Wenger, and D. S. Weiss, A quantum Newton's cradle, Nature 440, 900 (2006).

[42] S. Hofferberth, I. Lesanovsky, B. Fischer, T. Schumm, and J. Schmiedmayer, Non-equilibrium coherence dynamics in one-dimensional Bose gases, Nature 449, 324 (2007).

[43] S. Trotzky, Y.-A. Chen, A. Flesch, I. P. McCulloch, U. Schollwöck, J. Eisert, and I. Bloch, Probing the relaxation towards equilibrium in an isolated strongly correlated one-dimensional Bose gas, Nat. Phys. 8, 325 (2012).

[44] M. Cheneau, P. Barmettler, D. Poletti, M. Endres, P. Scbauß, T. Fukuhara, C. Gross, I. Bloch, C. Kollath, and S. Kuhr, Light-cone-like spreading of correlations in a quantum many-body system, Nature 481, 484 (2012).

[45] M. Gring, M. Kuhnert, T. Langen, T. Kitagawa, B. Rauer, M. Schreitl, I. Mazets, D. Adu Smith, E. Demler, and J. Schmiedmayer, Relaxation and Prethermalization in an Isolated Quantum System, Science 337, 1318 (2012).

[46] T. W. B. Kibble, Topology of cosmic domains and strings, J. Phys. A 9, 1387 (1976).

[47] W. H. Zurek, Cosmological experiments in superfluid helium?, Nature 317, 505 (1985).

[48] W. H. Zurek, U. Dorner, and P. Zoller, Dynamics of a quantum phase transition, Phys. Rev. Lett. 95, 105701 (2005).

[49] A. Polkovnikov and V. Gritsev, Breakdown of the adiabatic limit in low-dimensional gapless systems, Nature Phys. 4, 477 (2008).

[50] A. Chandran, A. Erez, S. S. Gubser, and S. L. Sondhi, Kibble-Zurek problem: Universality and the scaling limit, Phys. Rev. B 86, 064304 (2012).

[51] In particular, for $g=0.5, \eta(g)=1.0370(5)$ and $b(g) \approx$ 0.481 . For $g=0.8, \eta(g)=0.455(5)$ and $b(g) \approx 0.15[11$.

[52] In this work we have simulated quantum Ising chains in Eq. (1), with up to $L=22$ sites. The initial ground state has been obtained through Lanczos diagonalization, while for the time evolution we integrated the timedependent Schrödinger equation through a fourth-order Runge-Kutta method, with a time step $d t=10^{-3}$ sufficiently small to ensure convergence for all our purposes.

[53] C. Zener, Non-adiabatic crossing of energy levels, Proc. R. Soc. London, Ser A 137, 696 (1932); L. Landau, On the theory of transfer of energy at collisions II, Phys. Z. Sowjetunion 2, 46 (1932).

[54] N. V. Vitanov and B. M. Garraway, Landau-Zener model: Effects of finite coupling dynamics, Phys. Rev. A 53, 4288 (1996); N. V. Vitanov, Transition times in the Landau-Zener model, Phys. Rev. A 59, 988 (1999).

[55] M. Abramowitz and I. A. Stegun ed., Handbook of Mathematica Functions (Dover, New York, 1964).

[56] B. M. McCoy and T. T. Wu, Two-dimensional Ising field theory in a magnetic field: breakup of the cut in the two-point function, Phys. Rev. D 4, 1259 (1978).

[57] R. Coldea, D. A. Tennant, E. M. Wheeler, E. Wawrzynska, D. Prabhakaran, M. Telling, K. Habicht, P. Smeibidl, and K. Kiefer, Quantum criticality in an Ising chain: experimental evidence of the emergent $E_{8}$ symmetry, Science 327, 177 (2010).

[58] S. B. Rutkevich, On the weak confinement of kinks in the one-dimensional quantum ferromagnet $\mathrm{CoNb}_{2} \mathrm{O}_{6}, \mathrm{~J}$. Stat. Mech. (2010) P07015.

[59] J. Simon, W. S. Bakr, R. Ma, M. E. Tai, P. M. Preiss, and M. Greiner, Quantum simulation of antiferromagnetic spin chains in an optical lattice, Nature 472, 307 (2011).

[60] I. Bloch, Quantum coherence and entanglement with ultracold atoms in optical lattices, Nature 453, 1016 (2008).

[61] J. Simon, W. S. Bakr, R. Ma, M. E. Tai, P. M. Preiss, and M. Greiner, Quantum simulation of antiferromagnetic spin chains in an optical lattice, Nature 472, 307 (2011).

[62] K. Kim, M.-S. Chang, S. Korenblit, R. Islam, E. E. Edwards, J. K. Freericks, G.-D. Lin, L.-M. Duan, and C. Monroe, Quantum simulation of frustrated Ising spins with trapped ions, Nature 465, 590 (2010).

[63] E. E. Edwards, S. Korenblit, K. Kim, R. Islam, M.S. Chang, J. K. Freericks, G.-D. Lin, L.-M. Duan, and C. Monroe, Quantum simulation and phase diagram of the transverse-field Ising model with three atomic spins, Phys. Rev. B 82, 060412(R) (2010).

[64] R. Islam, E. E. Edwards, K. Kim, S. Korenblit, C. Noh, H. Carmichael, G.-D. Lin, L.-M. Duan, C.-C. Joseph 
Wang, J. K. Freericks, and C. Monroe, Onset of a quantum phase transition with a trapped ion quantum simulator, Nat. Commun. 2, 377 (2011).

[65] G.-D. Lin, C. Monroe, and L.-M. Duan, Sharp Phase Transitions in a Small Frustrated Network of Trapped Ion Spins, Phys. Rev. Lett. 106, 230402 (2011).

[66] K. Kim, S. Korenblit, R. Islam, E. E. Edwards, M.-S. Chang, C. Noh, H. Carmichael, G.-D. Lin, L.-M. Duan, C. C. Joseph Wang, J. K. Freericks, and C. Monroe, Quantum simulation of the transverse Ising model with trapped ions, New J. Phys. 13, 105003 (2011).

[67] P. Richerme, Z.-X. Gong, A. Lee, C. Senko, J. Smith, M. Foss-Feig, S. Michalakis, A. V. Gorshkov, and C. Monroe, Non-local propagation of correlations in long-range interacting quantum systems, Nature 511, 198 (2014).

[68] P. Jurcevic, B. P. Lanyon, P. Hauke, C. Hempel, P. Zoller,
R. Blatt, and C. F. Roos, Observation of entanglement propagation in a quantum many-body system, Nature 511, 202 (2014).

[69] S. Debnath, N. M. Linke, C. Figgatt, K. A. Landsman, K. Wright, and C. Monroe, Demonstration of a small programmable quantum computer with atomic qubits, Nature 536, 63 (2016).

[70] H. Labuhn, D. Barredo, S. Ravets, S. de Leseleuc, T. Macri, T. Lahaye, and A. Browaeys, Tunable twodimensional arrays of single Rydberg atoms for realizing quantum Ising models, Nature 534, 667 (2016).

[71] A. Keesling et al., Quantum Kibble-Zurek mechanism and critical dynamics on a programmable Rydberg simulator, Nature 568, 207 (2019). 